\title{
Central Serous Chorioretinopathy in Elderly Patients Mimicking Occult Neovascular Age-Related Macular Degeneration
}

This article was published in the following Dove Press journal: Clinical Ophthalmology

\author{
Sean D Adrean $\mathbb{D}^{1}$ \\ Siyang Chaili ${ }^{2}$ \\ Ash Pirouz' \\ Scott Grant ${ }^{\prime}$
}

'Retina Consultants of Orange County, Fullerton, CA 92835, USA; ${ }^{2}$ Vanderbilt Eye Institute, Vanderbilt University Medical Center, Nashville, TN 37232, USA
Correspondence: Sean D Adrean Retina Consultants of Orange County, 301 W. Bastanchury Ave \#285, Fullerton, CA 92835 , USA

Tel +|-7| $4-738-4620$

Email seadrean@yahoo.com
Purpose: To describe elderly patients with central serous chorioretinopathy (CSCR) mimicking occult neovascular age-related macular degeneration (nAMD).

Materials and Methods: The records of 522 patients with initial diagnoses of nAMD over one year were reviewed to determine characteristics meeting diagnostic criteria for CSCR with three or more months of follow-up. Patients were evaluated by clinical examination, fluorescein angiography (FA) and optical coherence tomography (OCT). At the time of initial evaluation, patients were either monitored, treated with anti-VEGF therapy or with combination anti-VEGF and photodynamic therapy (PDT). When no response to anti-vascular endothelial growth factor (anti-VEGF) treatment was observed, the diagnosis of CSCR was favored and patients were observed with close follow-up. The Student's $t$-test was used for statistical analysis; a p-value $<0.05$ was considered statistically significant.

Results: Eleven elderly patients met diagnostic criteria for CSCR among 522 patients initially diagnosed with nAMD. Average age was 75.9 years, and average follow-up was 16.9 months. Average presenting visual acuity was 20/50+2 (67.9 \pm 5.9 ETDRS letters), and choroidal thickness was $232.0 \pm 69.4 \mu \mathrm{m}$. After observation or treatment, the average vision improved to $20 / 40+(70.5 \pm 7.8$ ETDRS letters, $p=0.289)$. When intravitreal bevacizumab was given, no changes were observed for patients' neurosensory retinal detachments (NSRD). When NSRD changes were observed, they likewise did not correlate to the timing of anti-VEGF treatment. For patients who were monitored alone, one patient lost one line of vision, one gained one line, one gained two lines, and one gained three lines. One patient subsequently developed a choroidal neovascular membrane (CNVM) during initial follow-up with visual improvement after anti-VEGF treatment. Four patients developed CNVM overall with long term follow-up.

Conclusion: CSCR in elderly patients can mimic occult CNVM, especially on FA. In this group, many patients were monitored without treatment, which typically resulted in stable or improved vision. Careful monitoring is required because of possible development of CNVM.

Keywords: central serous chorioretinopathy, elderly, choroidal neovascular membranes, choroid

\section{Introduction}

Central serous chorioretinopathy (CSCR) is characterized by detachment of the neurosensory retina due to subretinal accumulation of fluid from the choriocapillaris. ${ }^{1}$ The pathogenesis of CSCR is not fully understood; however, implicated factors include stress, elevated cortisol levels, ${ }^{2}$ sleep apnea, hypertension, smoking, alcohol consumption, 
autoimmune disease, and retinal perfusion. ${ }^{3-5}$ CSCR is uncommon and has the highest prevalence in males in their forties, with an annual incidence of 10 per 100,000 in males, occurring less frequently in females, whereas AMD is much more common at 1500 per 100,000 people. ${ }^{1,6}$ While CSCR generally occurs in younger individuals, studies have found CSCR to occur in elderly patients, typically defined as patients older than 70 years old. ${ }^{2}$

The signs and symptoms of CSCR may resemble occult choroidal neovascularization (CNVM) due to agerelated macular degeneration (nAMD) and, thus, makes initial diagnosis challenging in routine practice. ${ }^{7,8}$ Patients may have blurred vision, a relative central scotoma, and metamorphopsia, which may wax and wane; and, visual acuity generally ranges from 20/20 to 20/200 at presentation. ${ }^{2}$ Specifically, patients may have leakage on FA and, thus, subretinal fluid demonstrated on OCT but without evidence intraretinal or subretinal hemorrhage on clinical exam or imaging. ${ }^{7-9}$ Distinguishing factors may include a pachychoroid, in which the choroid is thickened (>395 microns) and hyperpermeable, predisposing to patients to the condition. ${ }^{8,10,11}$ Patients may also exhibit a "double-layer sign", with RPE having an undulated appearance and Bruch's membrane that remains intact. ${ }^{12}$ Indocyanine green angiography (ICG) may show choroidal hyperpermeability, possibly due to binding of ICG molecule to fibrin, while fibrin is transparent on FA. ${ }^{7,8}$

Typically in routine practice, observation is attempted for CSCR and patients are told to discontinue corticosteroids as well as improve modifiable lifestyle factors such as alcohol and tobacco use. If no trend towards reduction in NSRD is observed after 2-3 months, treatment is considered to avoid damage to the photoreceptors. Thermal laser, micropulse laser and photodynamic therapy (PDT) have all been utilized. ${ }^{13,14}$ Unlike AMD, limited efficacy has been demonstrated in clinical trials for anti-vascular endothelial growth factor (anti-VEGF) agents in treating CSCR, unless the patient has subsequently developed CNVM as a complication. ${ }^{1}$ Prior retrospective studies have also suggested the role of mineralocorticoids in choroidal vasodilation and accumulation of subretinal fluid, although newer multicenter prospective studies have demonstrated this effect to be equivalent to placebo. $8,15,16$

This retrospective case series describes a cohort of elderly patients with CSCR in a retina practice mimicking occult nAMD and provides insight into the clinical decision process and visual outcomes following monitoring or treatment.

\section{Materials and Methods}

This retrospective study examined a series of elderly patients with visual loss, metamorphopsia and abnormal funduscopic exams in a clinical setting. The records of 522 patients initially diagnosed with nAMD in a retina practice were examined to determine the presence of CSCR in this elderly population. Visual acuity (VA) was evaluated using Snellen charts, and converted to Early Treatment Diabetic Retinopathy Study (ETDRS) letter equivalents for calculation of mean changes. SD-OCT (Heidelberg Engineering, Franklin, MA, USA) was used to measure central macular thickness (CMT) and choroidal thickness as able. Fluorescein angiography was used to visualize vascular leakage. In this population, certain patients had the appearance of occult CNVM on FA, but on SD-OCT there was only a NSRD without subretinal hemorrhage, intraretinal hemorrhage, or intraretinal fluid. Pigment epithelial detachments (PED) were also occasionally present. Patients with these findings were initially diagnosed with occult nAMD and some were initiated on anti-VEGF therapy (bevacizumab, Genentech, San Francisco, USA; ranibizumab, Genentech, San Francisco, USA). When there was little to no response to anti-VEGF therapy and patients had good presenting vision, the diagnosis of CSCR was favored. Patients were then carefully followed up as frequently as every two weeks initially, with longer separation of visits if vision was stable. Within a year, most patients were seen approximately quarterly. If patients developed CNVM, anti-VEGF therapy and PDT were employed as indicated.

The Student's $t$-test was performed for statistical analysis. Medical research involving human subjects was conducted according to the World Medical Association Declaration of Helsinki. Approval of this retrospective clinical series was performed by a local institutional review board (IRBCo; approval \#: 2017-0028-RCOC). Informed consent was waived since patient information was deidentified.

\section{Results}

The mean age of included patients ( 6 males and 5 females) was 75.9 years (range 65-91 years, Table 1). The average follow-up was 16.9 months (range, 2 months to 5 years). Patients were asked to adjust potential risk factors for CSCR as able. 
Table I Clinical Outcomes of Elderly Patients with CSCR

\begin{tabular}{|c|c|c|c|c|c|c|c|}
\hline $\begin{array}{l}\text { Patient } \\
\text { Number }\end{array}$ & Age & Sex & $\begin{array}{l}\text { Initial Vision } \\
\text { Snellen } \\
\text { (ETDRS Letter } \\
\text { Equiv.) }\end{array}$ & Treatment & $\begin{array}{l}\text { Duration of Follow-Up } \\
\text { (Months) }\end{array}$ & $\begin{array}{l}\text { Course of Disease } \\
\text { (Recurrence) }\end{array}$ & $\begin{array}{l}\text { Final Vision } \\
\text { Snellen } \\
\text { (ETDRS Letter } \\
\text { Equiv.) }\end{array}$ \\
\hline I & 82 & $\mathrm{~F}$ & $20 / 80(55)$ & None & 2 & None & $20 / 70(57)$ \\
\hline 2 & 91 & $F$ & $20 / 40(70)$ & PDT & 7 & Yes $x \mathrm{I}$ & $20 / 30(76)$ \\
\hline 3 & 65 & $\mathrm{~F}$ & $20 / 30(76)$ & None & 16 & Wax & $20 / 40(70)$ \\
\hline 4 & 75 & $F$ & $20 / 40(70)$ & BVZ & 18 & Wax & $20 / 60(6 I)$ \\
\hline 5 & 68 & $M$ & $20 / 50(65)$ & None & 13 & Yes $\times 1$ & $20 / 40(70)$ \\
\hline 6 & 79 & M & $20 / 30(76)$ & $B V Z$ & 60 & Yes $\times 2$ & $20 / 40(70)$ \\
\hline 7 & 75 & $M$ & $20 / 50(65)$ & None & 6 & None & $20 / 30(76)$ \\
\hline 8 & 85 & $M$ & $20 / 40(70)$ & PDT/BVZ & 20 & Yes $x \mathrm{I}$ & $20 / 40(70)$ \\
\hline 9 & 67 & $M$ & $20 / 40(70)$ & None & 11 & None & $20 / 20(85)$ \\
\hline 10 & 78 & $M$ & $20 / 50(65)$ & BVZ & 9 & Wax & $20 / 50(65)$ \\
\hline II & 71 & $M$ & $20 / 50(65)$ & PDT/BVZ & 24 & CNV & $20 / 30(76)$ \\
\hline
\end{tabular}

Abbreviations: M, male; F, female; PDT, photodynamic therapy; BVZ, bevacizumab; ETDRS Equiv., Early Treatment of Diabetic Retinopathy Study Equivalent.

All patients had a NSRD on SD-OCT without subretinal hemorrhage, intraretinal hemorrhage or intraretinal fluid on imaging or exam. Four patients showed drusen in their fellow eye while one patient had bilateral CSCR. Fluorescein angiograms of all patients had the appearance of an occult CNV. There were with multiple areas of pinpoint hyperfluorescence, corresponding to the area of the NSRD that became more prominent during the late phase of the FA. The mean CRT on OCT at baseline was $322.6 \pm 110.1 \mu \mathrm{m}$, while choroidal thickness was $232.0 \pm 69.4 \mu \mathrm{m}$. The choroidal thickness of patients' fellow eyes were on average $220.6 \pm 62.9 \mu \mathrm{m}$. Six of 11 patients (66.7\%) exhibited the double layer sign (an undulated RPE layer with an intact Bruch's membrane) while 7/11 (77.8\%) patients had a PED (Figure 1).
At presentation, the mean VA was $20 / 50+2(67.9 \pm 5.9$ ETDRS letters). Mean VA at the end of follow-up was 20/40 $+(70.5 \pm 7.8$ ETDRS $)$. The mean VA change from baseline was 2.6 letters $(\mathrm{p}=0.289)$. Overall, 5 patients improved by $\geq 1$ ETDRS line, 3 patients lost $\geq 1$ line and 3 had less than 1-line change in VA (Figure 2). Three subjects received bevacizumab alone; two of these patients lost $\geq 1$ line, while the other had no change in vision. OCT findings for these subjects generally did not change markedly (Figure 2); however, 2 subjects who were only monitored had clinically relevant reductions in CRT of 104 and $209 \mu \mathrm{m}$ at the end of followup with improvement in VA (5 and 15 letters, respectively).

Patients treated with intravitreal bevacizumab showed no initial change in their NSRD. When the NSRD changed, it
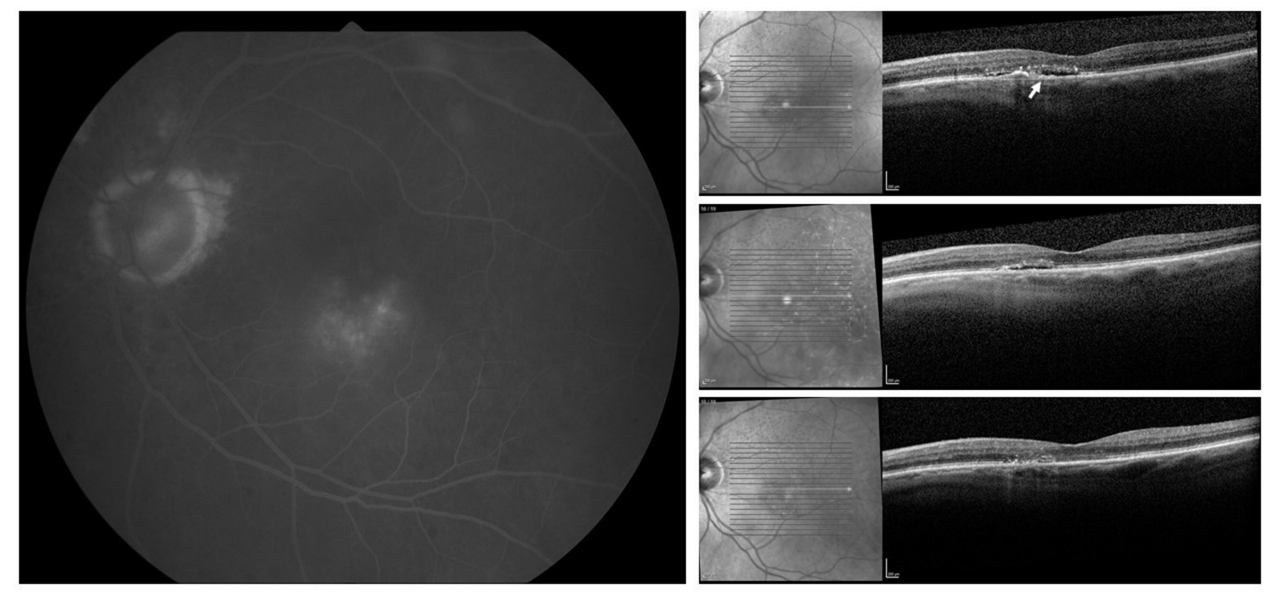

Figure I FA and OCT of CSCR in the Elderly. Left: Representative late FA in Patient 6 demonstrating occult like CNVM in a patient with CSCR of the elderly. Right, top to bottom: Patient 5 with sequential SD-OCT imaging showing CSCR that resolved over time, without intervention. This patient had a PED and exhibited the DLS (yellow arrow). 

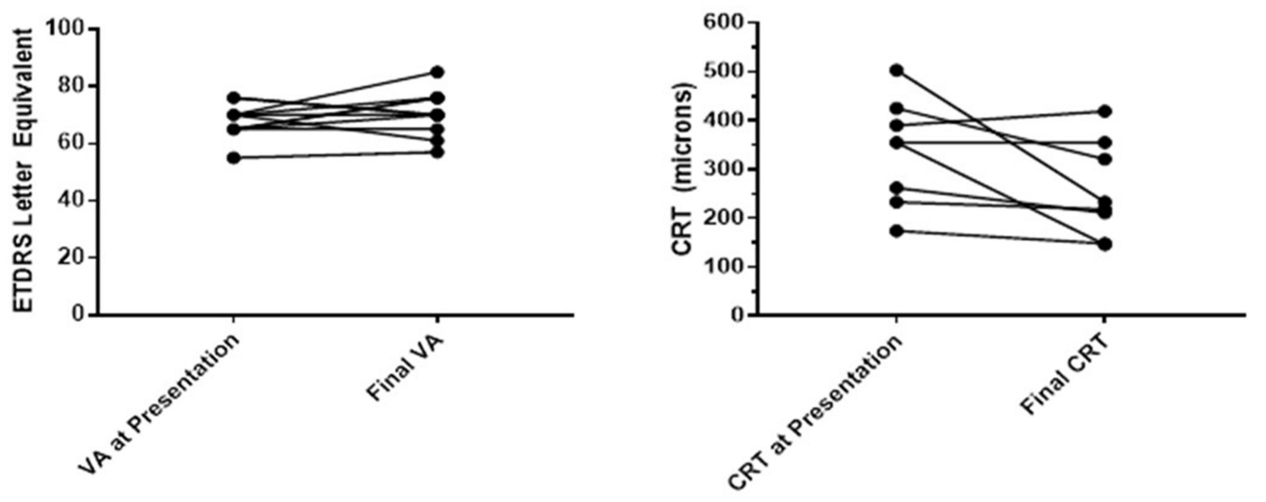

Figure 2 Scatter plot of visual acuity change during the study. Visual acuity (VA) reported in ETDRS letter equivalents at presentation and end of data collection demonstrated that most subjects maintained or improved VA during the study period. The central retinal thickness in all but 2 subjects maintained or reduced during the study period.

did not correlate with the timing of the intravitreal injections. Two patients received combination of PDT and bevacizumab. Of these, one subject had converted to CNVM with evidence of retinal hemorrhage on biomicroscopy and OCT, but with treatment improved by 11 letters. The other patient had no change in vision. The patient receiving PDT alone improved by 6 letters at the end of follow-up. For patients who were monitored alone, one patient lost one line of vision, one gained one line, one gained two lines, and one gained three lines. During total follow-up time, three patients had a single recurrence of the CSR, while one patient had two recurrences, for a total rate of recurrence of $44 \%$.

During this study period, 522 elderly patients were diagnosed with nAMD and 11 of these patients met diagnostic criteria for CSCR. Thus, the incidence of CSCR among elderly patients with initial symptoms similar to those of nAMD in our practice was $2.1 \%$.

\section{Discussion}

This retrospective case series demonstrates that CSCR occurs in elderly patients and may resemble occult neovascular AMD, although the disease entities are distinct. Elderly patients who present with new symptoms of decreased vision and metamorphopsia are more likely to be initially diagnosed with nAMD with initiation of antiVEGF therapy. The incidence of AMD in this population is greater at baseline. This may also be due to CSCR and its spectrum of diseases having features of Type 1 neovascularization and choroidal thickening, although some of the features of classic macular degeneration, such as drusen, may be absent. ${ }^{8}$ If an elderly patient presents with several months of metamorphopsia, relatively good vision, and a NSRD with possible PED, however, without intraretinal or subretinal blood, then CSCR of the elderly should be included as a likely differential diagnosis and close monitoring without treatment may be considered.

The decision to initially treat many of these patients as diagnosed with nAMD, rather than monitor the patients' condition, was based on several factors. First, given that in the elderly population nAMD is much 15 times more prevalent than CSCR, initial suspicion for nAMD should be higher. When patients presented with new symptoms of decreased vision and metamorphopsia, and had subsequent appearance of an occult CNVM on FA, initiation of antiVEGF therapy was started to minimize the risk of vision loss. Yet, patients typically presented with relatively good initial vision, likely because they had a shallow NSRD so photoreceptors may still be adequately nourished by the choroid. Some patients had noticeable distortions in their vision, but many presented with a more indolent and subtle course. Biomicroscopic findings showed a lack of intraretinal or subretinal hemorrhage and OCT demonstrated NSRD with possible PED. Likewise, intraretinal blood was not seen on OCT. Other modalities, such as OCTangiography (OCT-A) may be obtained to determine the presence of CNV lesions within the NSRD or choroid to better guide treatment; and if present, these patients should receive anti-VEGF therapy. Several challenges, however, may arise when utilizing this imaging modality such as poor signal strength and artefactual movement. Likewise, ICG, may aid in evaluation by demonstrating a hyperpermeable (pachy)choroid. Thus, elderly patients with decreased presenting vision, metamorphopsia, subretinal fluid on OCT, and leakage on FA might be initially thought to have occult nAMD. However, close attention to the lack of intraretinal or subretinal hemorrhage, on OCT or 
biomicroscopy may better fit the diagnosis of CSCR even in the elderly patient. OCT-A and ICG may also differentiate CSCR from occult nAMD in these patients. Interestingly, these patients did not have a pachychoroid (choroidal thickness $\geq 395$ microns), likely because as patients age choroidal thickness decreases. ${ }^{17,18}$ However, $66.7 \%$ of patients exhibited a DLS, which is consistent with other studies showing a DLS in $71.2 \%$ to $75 \%$ of patients with CSCR. ${ }^{12,19}$

Given that the incidence of nAMD in the elderly population is much greater than CSCR and that these patients presented with what appeared to be occult nAMD on exam, anti-VEGF therapy was the initial treatment of choice. If the subretinal fluid was not reduced by antiVEGF therapy and subretinal or intraretinal blood was not observed at any time point during the 2-3 month followup period then the diagnosis of CSCR of the elderly was favored, and anti-VEGF treatment was discontinued. Patients were closely monitored at this point. In most cases, vision improved over the course of months without treatment. Anti-VEGF had no discernable effect on subretinal fluid alone and when the amount of fluid changed it did not correlate with the timing of anti-VEGF treatment. Had this been nAMD, we would have expected that delivery of anti-VEGF would have correlated directly with decrease of fluid on exam and improvement of vision. This method was sufficient to diagnose patient's with CSCR; and, although OCT-A and ICG would have further aided in diagnosis, we believe it is not a requirement for the diagnosis of CSCR. If a patient subsequently developed a CNVM with evidence of intra- or subretinalbleeding or neovascular vessels/membrane, treatment with anti-VEGF agents was initiated. After the data collection period for this study and during longer-term followup, 3 additional subjects converted to CNVM at 22, 38 and 39 months. Thus, a total of $44.4 \%$ of patients (4/11) converted to CNVM. Therefore, long-term follow-up and evaluations are required. PDT was also considered if patients' VA failed to improve or worsened due to CSCR. Our results agree with Wong et al who reviewed the evidence for the treatments for CSCR and found that bevacizumab was not temporally associated with VA improvements while PDT was associated was. ${ }^{1}$ Overall, the vision in this cohort of patients did not significantly change over the course of the study. This may be due to the fact that patients overall had good presenting vision. Once a decision was made to treat, PDT was chosen as the treatment of choice, since all leakage was subfoveal and the appearance was more like occult CNVM; and, the entire area was treated with half-fluence PDT. There is convincing evidence supporting the safety and efficacy of half-fluence PDT as a first-line therapy. Maruko et al have shown with enhanced depth imaging OCT that the choroidal thickness was reduced 4 weeks after half-dose PDT, and indocyanine green angiography indicated a reduction in hyperpermeability. ${ }^{15}$ Micropulse laser may also be employed although none of the patients in this series underwent this treatment modality since none had "hot spots" that would allow for focal laser treatment.

Limitations of this study include its retrospective nature and relatively small sample size, however, it is known that the incidence of CSCR is much less than nAMD and likely even more so in this elderly population. Given that these patients initially were thought to have nAMD and were only found to have evidence which favored the diagnosis of CSCR after failure of initial anti-VEGF treatment, this study is inherently retrospective. The research in this area is currently expanding, and additional studies employing different diagnostic and treatment modalities evaluated in a prospective manner will continue to improve our practice. Still, this study provides insights into routine clinical practice where elderly patients with CSCR may be incorrectly diagnosed at first presentation with occult nAMD.

\section{Conclusion}

CSCR in elderly patients can mimic occult nAMD, especially on FA. In this population of patients initially diagnosed with nAMD, 2.1\% (11/522) of patients had disease that was ultimately more consistent with CSCR; the rest had exudative AMD which responded as anticipated to anti-VEGF therapy. CSCR of the elderly may be considered in those patients that have poor or no response to antiVEGF agents with resolution of NSRD at intervals not attributable to anti-VEGF therapy. Historical factors, good initial vision, and exam findings, including the absence of retinal hemorrhage, may help to establish the diagnosis. The disease typically improved with monitoring, however careful follow-up is required due to possible conversion to CNVM.

\section{Acknowledgments}

The authors would like to thank Frances Kane for assistance with preparation, writing and editing of this manuscript. 


\section{Disclosure}

Sean Adrean reports grants from Regeneron, Apellis, and Gemini and grants and personal fees from Allergan and Genentech, outside the submitted work. The author reports no other potential conflicts of interest in this work.

\section{References}

1. Wong KH, Lau KP, Chhablani J, Tao Y, Li Q, Wong IY. Central serous chorioretinopathy: what we have learnt so far. Acta Ophthalmol. 2016;97(4):321-325. doi:10.1111/aos.12779

2. Wang M, Munch IC, Hasler PW. Central serous chorioretinopathy. Acta Ophthalmol. 2008;86(2):126-145. doi:10.1111/j.16000420.2007.00889.x

3. Chatziralli I, Kabanarou SA, Parikakis E, et al. Risk factors for central serous chorioretinopathy: multivariate approach in a case-control study. Curr Eye Res. 2017;42(7):1069-1073.

4. Caccavale A, Romanazzi F, Imparato M, Negri A. Central serious chorioretinopathy: a pathogenetic model. Clin Ophthalmol. 2011;5:239-243. doi:10.2147/OPTH.S17182

5. Moschos M, Nitoda E. Pathophysiology of visual disorders induced by phosphodiesterase inhibitors in the treatment of erectile dysfunction. Drug Des Devel Ther. 2016;10:3407-3413. doi:10.2147/DDDT. S118015

6. Leibowitz H, Krueger DE, Maunder LR, et al. The framingham eye study monograph. Surv Ophthalmol. 1980;24:335-610.

7. Spaide RF, Hall L, Haas A, et al. Indocyanine green videoangiography of older patients with central serous chorioretinopathy. Retina. 1996;16 (3):203-213. doi:10.1097/00006982-199616030-00004

8. Pang CE, Bailey-Freund K. Pachychoroid neovasculopathy. Retina. 2015;35(1):1-9. doi:10.1097/IAE.0000000000000331

9. Daruich A, Metet A, Dirani A, et al. Central serous chorioretinopathy: recent findings and new physiopathology hypothesis. Prog Retin Eye Res. 2015;48:82-118. doi:10.1016/j.preteyeres.2015.05.003
10. Adhi M, Duker JS. Optical coherence tomography - current and future applications. Curr Opin Ophthalmol. 2013;24(3):213-221. doi:10.1097/ICU.0b013e32835f8bf8

11. Manayath GJ, Shah VS, Saravanan VR, Narendran V. Polypoidal choroidal vasculopathy associated with central serous chorioretinopathy: pachychoroid spectrum of diseases. Retina. 2017. doi:10.1097/ IAE.00000000000001665

12. Yang L, Jonas JB, Wei W. Optical coherence tomography-assisted enhanced depth imaging of central serous chorioretinopathy. Invest Ophthalmol Vis Sci. 2013;54(7):4659-4665. doi:10.1167/iovs.1210991.

13. Dhirani NA, Yang Y, Somani S. Long-term outcomes in half-dose verteporfin photodynamic therapy for chronic central serous retinopathy. Clin Ophthalmol. 2017;6(11):2145-2149. doi:10.2147/ OPTH.S151933

14. Scholz P, Altay L, Fauser S. A review of subthreshold micropulse laser for treatment of macular disorders. Adv Ther. 2017;45 (7):1528-1555. doi:10.1007/s12325-017-0559-y

15. Yang D, Eliott D. Systemic mineralocorticoid antagonists in the treatment of central serous chorioretinopathy. Semin Ophthalmol. 2017;32(1):36-42. doi:10.1080/08820538.2016.1228418

16. Schwartz R, Habot-Wilner Z, Martinez MR, et al. Eplerenone for chronic central serous chorioretinopathy-a randomized controlled prospective study. Acta Ophthalmol. 2017;95(7):e610-e618. doi:10.1111/aos.13491

17. Maruko I, Iida T, Oyamada H, Sugano Y, Ojima A, Sekiryu T. Choroidal thickness changes after intravitreal ranibizumab and photodynamic therapy in recurrent polypoidal choroidal vasculopathy. $\mathrm{Am}$ J Ophthalmol. 2013;156(3):548-556. doi:10.1016/j.ajo.2013.03.041

18. Spaide RF. Age-related choroidal atrophy. Am J Ophthalmol. 2009;147(5):801-810. doi:10.1016/j.ajo.2008.12.010

19. Lehmann M, Bousquet E, Beydoun T, Behar-Cohen F. Pachychoroid: an inherited condition? Retina. 2015;35(1):10-16. doi:10.1097/ IAE.0000000000000287
Clinical Ophthalmology

\section{Publish your work in this journal}

Clinical Ophthalmology is an international, peer-reviewed journal covering all subspecialties within ophthalmology. Key topics include: Optometry; Visual science; Pharmacology and drug therapy in eye diseases; Basic Sciences; Primary and Secondary eye care; Patient Safety and Quality of Care Improvements. This journal is indexed on PubMed

\section{Dovepress}

Central and CAS, and is the official journal of The Society of Clinical Ophthalmology (SCO). The manuscript management system is completely online and includes a very quick and fair peer-review system, which is all easy to use. Visit http://www.dovepress.com/ testimonials.php to read real quotes from published authors. 